\title{
Entéropathies congénitales et transplantation intestinale
}

\section{Olivier Goulet}

Centre de référence pour les maladies digestives rares, Service de gastro-entérologie, hépatologie et nutrition pédiatriques, Hôpital Necker - Enfants Malades, Paris, France

\section{Mots-clés \\ Insuffisance intestinale $\cdot$ Citrulline $\cdot$ Entéropathie congénitale $\cdot$ Nutrition parentérale $\cdot$ Hépatopathie $\cdot$ Transplantation intestinale $\cdot$ Transplantation hépatique $\cdot$ Nutrition parentérale à domicile}

\section{Résumé}

Une insuffisance intestinale nécessite une nutrition parentérale. Les cas d'insuffisance intestinale sévère prolongée incluent le syndrome du grêle court, des troubles sévères de la motilité (aganglionnose totale ou subtotale ou syndrome de pseudo-occlusion intestinale chronique) et des anomalies congénitales du développement des entérocytes. Une hépatopathie sévère peut se développer chez les patients atteints d'une insuffisance intestinale tant en raison de l'affection digestive sousjacente que d'une nutrition parentérale inadaptée. Une septicémie liée au cathéter et/ou une thrombose vasculaire étendue peuvent nuire à la poursuite d'une nutrition parentérale sûre et efficace. La prise en charge des patients atteints d'une insuffisance intestinale nécessite donc l'identification précoce de l'affection et l'analyse de son risque d'irréversibilité. La détermination du moment auquel adresser le patient pour une transplantation intestinale demeure un problème capital. En conséquence, la prise en charge doit inclure des traitements adaptés à chaque stade d'insuffisance intestinale sur la base d'une approche multidisciplinaire dans des centres assurant des soins de gastro-entérologie pédiatrique, une expertise en nutrition parentérale, un programme de nutrition parentérale à domicile, des interventions de chirurgie pédiatrique et un programme de transplantation hépatointestinale.

Copyright (๑) 2006 Nestec Ltd., Vevey/S. Karger AG, Basel

L'insuffisance intestinale définit un état de malabsorption intestinale sévère nécessitant une nutrition parentérale. Les entéropathies congénitales qui altèrent la muqueuse intestinale aboutissent à une insuffisance intestinale sévère. Le risque de complications d'une nutrition parentérale permanente est élevé, ce qui soulève la question de la nécessité éventuelle d'une transplantation intestinale.

\section{Définition de l'insuffisance intestinale}

L'insuffisance intestinale se définit par la réduction critique de la masse intestinale fonctionnelle au-dessous de la quantité minimale nécessaire à une absorption et une digestion adéquates pour la satisfaction des besoins nutritionnels et liquidiens de l'organisme indispensable

\section{KARGER}

Fax +41613061234

E-Mail karger@karger.ch

www.karger.com
(C) 2006 Nestec Ltd., Vevey/S. Karger AG, Base 0250-9644/06/0641-0025\$23.50/0

Accessible en ligne à www.karger.com/anf
Olivier Goulet

Reference Center for Rare Digestive Diseases, Hôpital Necker - Enfants Malades 149 Rue de Sèvres

FR-75743 Paris Cedex 15 (France)

Tel. +331444944 11, Fax +331444925 01, E-Mail olivier.goulet@nck.aphp.fr 
au maintien de la santé chez l'adulte et à la croissance chez l'enfant. Cette définition n'est cependant pas universellement acceptée. Certains auteurs suggèrent que l'insuffisance intestinale est mieux définie en terme de perte fécale d'énergie que de longueur intestinale résiduelle chez les patients présentant un syndrome du grêle court [1]. Une autre approche consiste à déterminer le degré d'insuffisance intestinale en fonction de la quantité de nutrition parentérale nécessaire au maintien de la santé chez l'adulte et à la croissance chez l'enfant. En sus des critères anatomiques et nutritionnels de définition de l'insuffisance intestinale, un marqueur biologique de la masse intestinale fonctionnelle a été récemment proposé [2]. La citrulline est un acide aminé non essentiel principalement produit par les entérocytes [3]. La concentration plasmatique en citrulline peut être facilement mesurée et sa diminution traduit la perte de masse fonctionnelle chez les patients atteints d'un syndrome du grêle court ou présentant divers degrés d'atrophie villositaire ou un rejet de greffe intestinale avec atrophie villositaire [4]. Une récente étude menée chez des patients pédiatriques atteints d'un syndrome du grêle court traité par hormone de croissance humaine recombinante a montré que la concentration plasmatique en citrulline augmentait significativement à la suite du traitement [5]. Le critère actuel définissant l'insuffisance intestinale demeure l'inaptitude à préserver la croissance d'un enfant sans dépendance permanente de la nutrition parentérale.

\section{Causes de l'insuffisance intestinale}

L'histoire naturelle de l'insuffisance intestinale chez l'enfant est largement influencée par l'anomalie sousjacente responsable de la perte de la fonction intestinale. Le type le plus fréquent d'insuffisance intestinale est secondaire à une infection digestive. Sa guérison totale est alors la règle avec un traitement approprié. Une diarrhée prolongée post-entéritique demeure cependant une des principales causes d'insuffisance intestinale dans les pays en développement [6]. Dans les pays industrialisés, l'insuffisance intestinale est dominée par l'apparition congénitale ou précoce de manifestations pathologiques sévères et permanentes nécessitant une nutrition parentérale prolongée ou de durée indéterminée. Historiquement, le syndrome du grêle court a été l'une des premières causes reconnues d'insuffisance intestinale prolongée [7]. Cependant, l'emploi croissant et l'éfficacité de la nutrition parentérale à long terme ont permis l'émergence ces trois dernières décennies de plusieurs autres causes d'insuffisance intestinale, par exemple des troubles sévères et étendus de la motricité dont l'aganglionnose totale, le syndrome de pseudo-inclusion intestinale chronique et les anomalies congénitales du développement des entérocytes [8].

\section{Entéropathies congénitales responsables d'une insuffisance intestinale}

Une tentative de classification des diarrhées intraitables du nourrisson en fonction du degré d'atrophie villositaire a été proposée sur la base de critères immunohistochimiques soulignant le rôle des lymphocytes activés dans la muqueuse intestinale [9]. Une enquête multicentrique de l'ESPGHAN a recueilli des cas de diarrhées infantiles intraitables et d'atrophie villositaire dont les caractéristiques bien définies en microscopie optique ont permis le classement [10]. Le premier type a été caractérisé par une infiltration de la lamina propria par des cellules mononucléées et considéré comme associé à des lymphocytes T activés. Le second type histologique inclut la survenue précoce d'une diarrhée sévère avec atrophie villositaire sans infiltration de la lamina propria par des cellules mononucléées et des anomalies histologiques spécifiques affectant l'épithélium. Plusieurs types d'anomalies épithéliales primitives induisant une diarrhée intraitable du nourrisson ont été identifiés à ce jour. Le premier décrit a été l'atrophie microvillositaire ou maladie des inclusions microvillositaires, suivi plus récemment de la dysplasie épithéliale intestinale [11]. Des anomalies phénotypiques sont présentes chez certains nourrissons ayant une diarrhée intraitable et un retard de croissance intrautérin [12].

\section{Atrophie microvillositaire}

En 1978, Davidson et coll. [13] ont publié les cas de cinq nourrissons présentant une diarrhée persistante sévère ayant débuté au cours de la période néonatale et chez lesquels la microscopie optique avait révélé une atrophie villositaire avec hypoplasie des cryptes. L'examen en microscopie électronique de biopsies de l'intestin grêle de trois des patients a révélé des anomalies sévères de la bordure en brosse et une augmentation de corpuscules ressemblant à des liposomes, et, chez l'un d'entre eux, des inclusions intracytoplasmiques ayant une structure de bordure en brosse retrouvées dans d'autres séries [14]. Ces descriptions cliniques et histologiques ont permis d'établir que l'atrophie microvillositaire était une entité distincte dans le cadre des diarrhées intraitables du nour- 
risson. Dans sa forme typique, il s'agit d'une affection congénitale des cellules épithéliales intestinales qui se présente initialement comme une diarrhée néonatale intraitable [14].

\section{Expression clinique}

En général, les nourrissons atteints présentent une diarrhée aqueuse sévère au cours des premiers jours suivant la naissance. Le débit fécal peut être très élevé (jusqu'à 250-300 $\mathrm{ml}$ par $\mathrm{kg}$ de poids et par jour) et les concentrations fécales en électrolytes sont voisines de celles du contenu de l'intestin grêle. Ces troubles menacent le pronostic vital, car une diarrhée massive entraîne une déshydratation rapide, des déséquilibres électrolytiques et ultérieurement une décompensation métabolique en quelques heures. La diarrhée aqueuse sévère persiste malgré le repos intestinal. Le diagnostic différentiel peut être une diarrhée chlorée congénitale ou une diarrhée par malabsorption du sodium, qui peuvent être précocement différenciées d'une atrophie microvillositaire par la mesure des ionogrammes sanguin et fécal [15-16]. En revanche, la présentation clinique d'une atrophie microvillositaire peut être également très atypique, avec un intestin grêle et un côlon pleins et distendus, simulant un syndrome de pseudo-obstruction. La diarrhée due à une atrophie microvillositaire survient le plus souvent au cours des tout premiers jours de la vie (début précoce ou forme congénitale). La diarrhée peut toutefois apparaître de façon retardée (premier mois) et moins sévère (forme à début tardif).

Analyses anatomopathologiques

Le diagnostic peut être facilement porté à l'examen en microscopie optique de biopsies duodénales ou jéjunales. A la coloration à l'hématoxyline-éosine, la muqueuse apparaît aplatie et présente une atrophie villositaire hypoplasique. L'accumulation de granules sécrétoires prenant la coloration PAS (periodic acid-Schiff) dans le cytoplasme apical des entérocytes est très caractéristique de cette affection [17, 18] (fig. 1). En microscopie électronique on observe des microvillosités rares ou absentes et des inclusions microvillositaires dans le cytoplasme des entérocytes. Ces modifications anatomiques définissent cette entité [19]. Une nouvelle technique utile de diagnostic de l'atrophie microvillositaire en microscopie optique a été récemment proposée [20, 21]. Le CD-10, peptidase neutre associée à la membrane, marque de façon linéaire la bordure en brosse de l'intestin grêle. Contrairement à ce marquage de surface dans différentes situations (intestin normal, maladie coeliaque, entéropa- thie auto-immune et allergie), tous les cas d'atrophie microvillositaire présentent une immunoréactivité CD10 intracytoplasmique importante dans les entérocytes des villosités [20]. Des résultats semblables ont été obtenus avec le PAS, l'antigène carcinoembryonnaire polyclonal et la phosphatase alcaline, trois marqueurs connus pour réaliser le marquage cytoplasmique des entérocytes superficiels dans l'atrophie microvillositaire [22]. En microscopie électronique, les cellules épithéliales des villosités n'ont pas de microvillosités, ou des microvillosités manifestement anormales, et contiennent de nombreux corps vésiculaires de taille variable et des inclusions microvillositaires caractéristiques (fig. 2). La morphologie des cellules cryptiques est quasiment normale. Elles ne contiennent pas un nombre accru de vésicules apicales ni de corps vésiculaires. Des inclusions microvillositaires et des granules sécrétoires en nombre accru sont également visibles dans l'épithélium colique. Ceci est particulièrement important, car ce tissu est plus facilement accessible pour une biopsie, particulièrement chez le petit nourrisson.

Physiopathologie et mode de transmission

Une anomalie du trafic membranaire des entérocytes immatures et/ou en cours de différenciation a été discutée comme mécanisme étiopathogénique de l'atrophie microvillositaire $[19,22]$. Une conséquence fonctionnelle directe de cette anomalie membranaire est une insuffisance intestinale totale. Selon une autre hypothèse, cette maladie serait associée à des perturbations du cytosquelette de la bordure en brosse des entérocytes dont l'assemblage serait anormal. Une dystrophie microvillositaire intestinale a été décrite à titre de variante hypothétique de l'atrophie microvillositaire [22]. La pathogenie de l'atrophie microvillositaire demeure toutefois mal élucidée. L'analyse du renouvellement de la sucrase-isomaltase, représentative des proteines de la bordure en brosse, montre clairement que les voies constitutives directes et indirectes du trafic membranaire sont intactes dans l'atrophie microvillositaire [19]. Etant donné que, tant dans la forme congénitale de l'atrophie microvillositaire que dans celle à début tardif, l'aspect des microvillosités est quasiment normal dans les régions où les granules sécrétoires s'accumulent initialement, une anomalie de la biosynthèse des microvillosités n'est probablement pas à l'origine de la maladie. A la suite de l'étude des granules sécrétoires, Phillips et coll. [23], ont récemment suggéré que l'atrophie microvillositaire comportât une anomalie de l'exocytose du glycocalyx qui pourrait altérer les fonctions cellulaires normales. 
Fig. 1. Coloration PAS (Periodic acidSchiff). A Muqueuse normale, PAS normal, coloration de la bordure en brosse. B Accumulation anormale du PAS, matériel positif dans le cytoplasme apical des cellules épithéliales dans une maladie des inclusions microvillositaires.
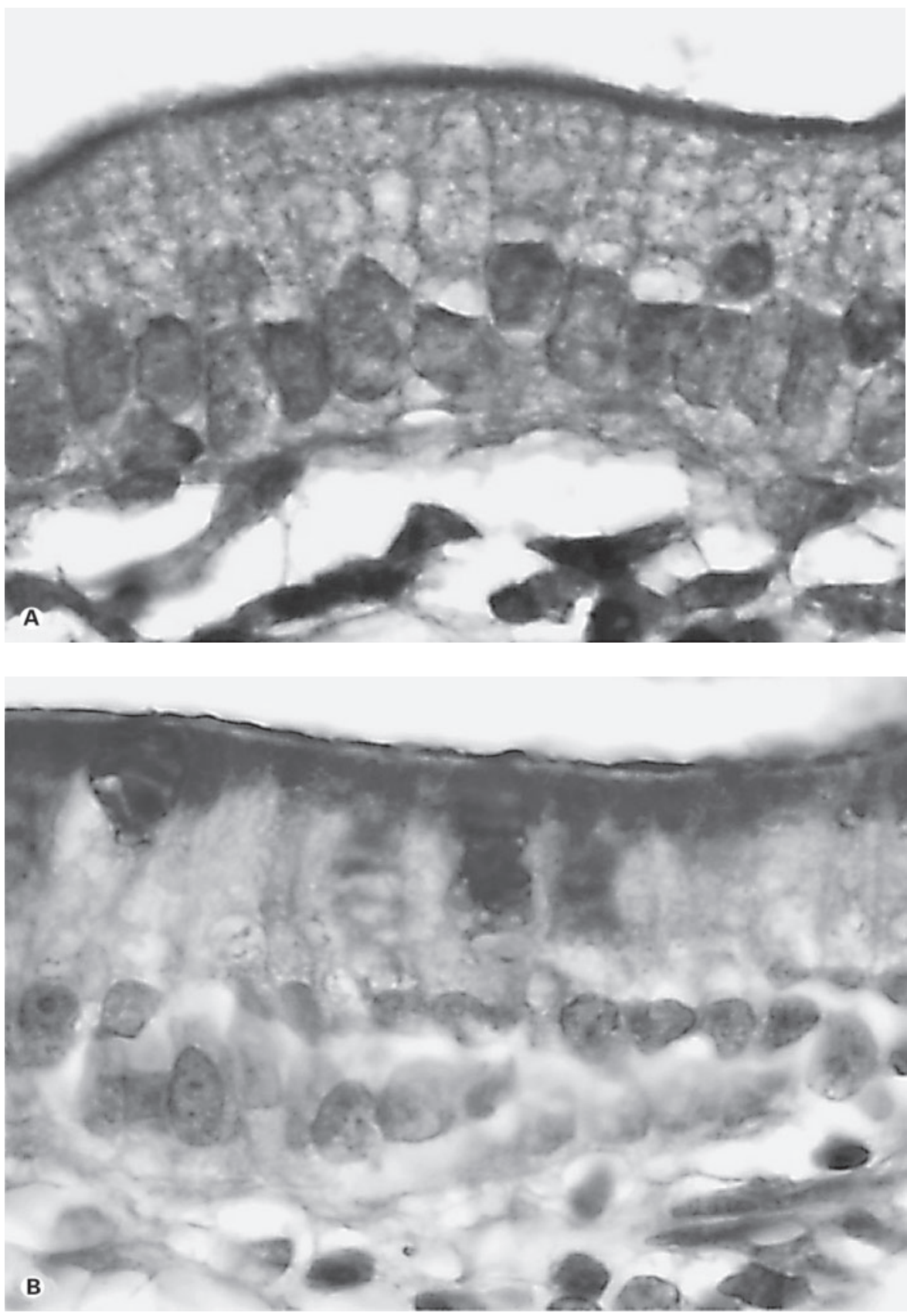

Compte tenu du nombre de cas de membres atteints dans une même fratrie et de la fréquence de la consanguinité parentale, cette maladie paraît être transmise comme un trait récessif autosomique [24]. Aucun gène candidat n'a été identifié à ce jour, mais une atrophie microvillositaire a été décrite chez une petite fille présentant une hypochondroplasie autosomique dominante [25]. L'anomalie génique liée à cette hypochondroplasie a été récem- ment localisée dans la région chromosomique 4 p16.3, ce qui pourrait faciliter l'élucidation de l'anomalie génétique de l'atrophie microvillositaire.

\section{Évolution à long terme}

L'enquête multicentrique la plus importante, menée chez 23 patients atteints d'une atrophie microvillositaire [14] a révélé une réduction extrême de l'espérance de vie 
Fig. 2. Maladie des inclusions microvillositaires. Microscopie électronique d'un échantillon de biopsie jéjunale. La bordure en brosse est quasiment absente. Le cytoplasme contient une inclusion microvillositaire.

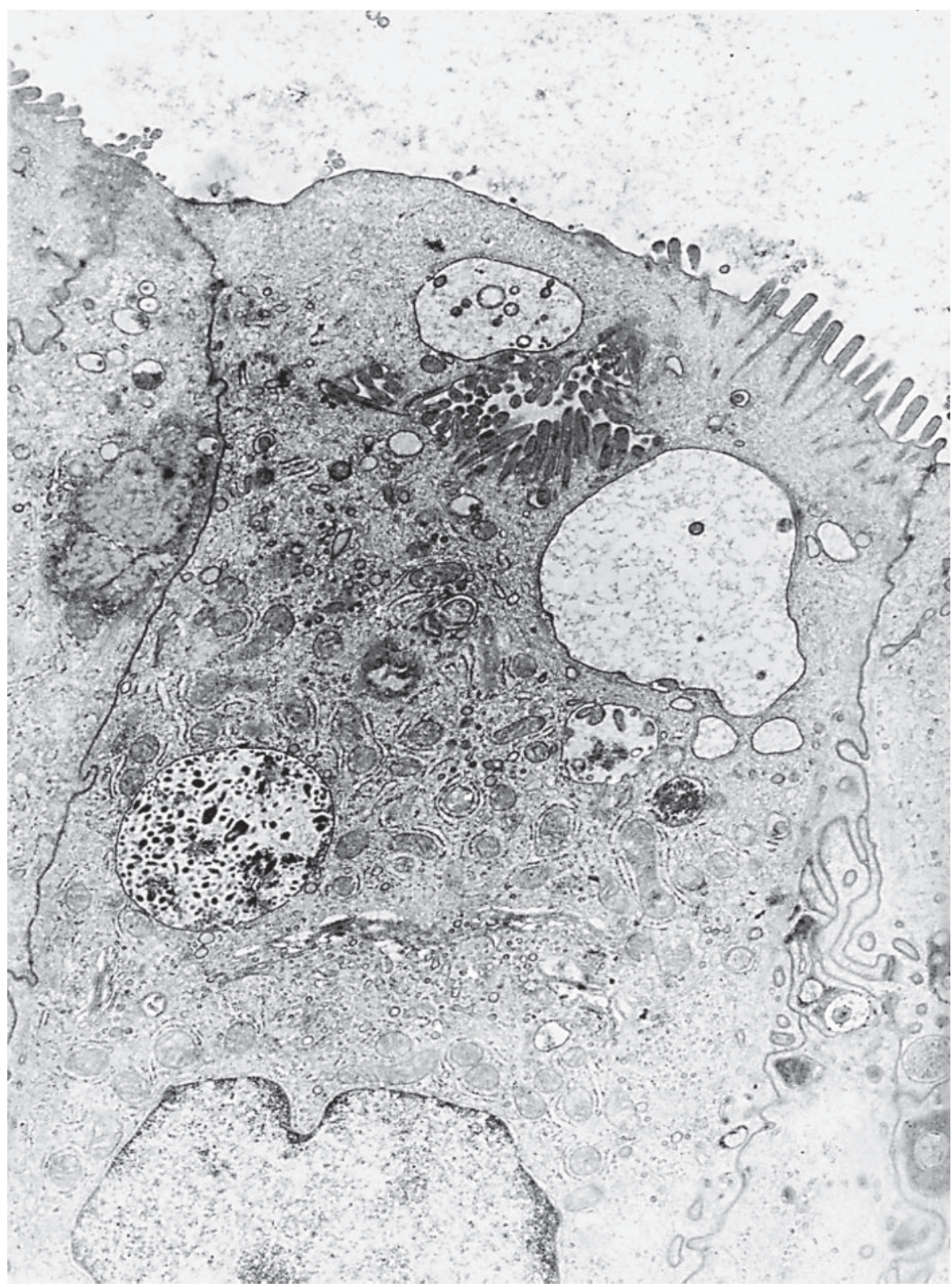

avec un taux de survie à un an inférieur à $25 \%$. La plupart des enfants décèdent de complications septiques, d'une insuffisance hépatique ou d'une décompensation métabolique. Quelques patients atteints d'une atrophie microvillositaire, particulièrement à début tardif et ceux chez lesquels le débit fécal n'est pas trop important peuvent survivre avec une nutrition parentérale partielle. Des tentatives thérapeutiques par corticoïdes, colostrum ou facteur de croissance épidermique n'ont pas été efficaces, mais l'octréotide a été utilisé avec un succès partiel chez un patient [14]. Contrairement à l'évolution initialement observée avant les années 1980, la nutrition parentérale autorise aujourd'hui la survie de la plupart des nourrissons. Des complications liées à une nutrition parentérale inadéquate limitent toutefois la survie à long terme. De plus, sans signe de néphropathie associée, certains de ces nourrissons et enfants présentent un déséquilibre hydroélectrolytique et une acidose chronique avec un retard de croissance secondaire. Certains autres sont atteints d'une néphrocalcinose en raison d'épisodes réitérés de déshydratation associés à des apports phosphocalciques inappropriés. Enfin, même avec une nutrition parentérale à 
long terme adéquate et une croissance normale, un débit fécal élevé et inconfortable persiste et nécessite souvent une substitution hydroélectrolytique en raison du risque élevé de déshydratation sévère. De ce fait, la transplantation intestinale peut être le seul traitement définitif de cette maladie intestinale rare.

Plusieurs cas de transplantation réussie ont été décrits dans des cas d'atrophie microvillositaire [26-29]. Il s'est agit de transplantations soit uniquement de l'intestin [26] soit en association à une transplantation hépatique [27, 28]. La nécessité de transplanter ou non le côlon avec l'intestin grêle demeure controversée. Nous avons récemment décrit les cas de patients présentant une atrophie microvillositaire congénitale à début précoce [29]. Deux patients sont décédés avant de pouvoir être inscrits sur une liste d'attente pour une transplantation de l'intestin grêle et un l'attend encore. Nous avons effectué une transplantation d'intestin de cadavre chez 7 patients âgés de 3 à 11 ans en utilisant le tacrolimus, des corticoïdes et des antagonistes de l'IL-2. Trois transplantations de l'intestin seul et quatre de l'intestin et du foie ont été réalisées. Une transplantation du côlon droit a été effectuée dans 5 cas (deux transplantations intestinales isolées). Six patients (86\%) ont survécu pendant une durée médiane de 3 ans (extrêmes: 1 et 8 ans). Un sevrage total de la nutrition parentérale a été obtenu chez tous les patients. Pour la première fois, la transplantation intestinale seule ou en association à celle du foie a offert à des enfants atteints d'une atrophie microvillositaire une perspective à long terme $[29,30]$.

\section{Dysplasie intestinale épithéliale}

Trois cas de diarrhée néonatale sévère avec images épithéliales anormales ont été décrits par Reifen et coll. [31] sous le nom de «tufting enteropathy» («entéropathie à houpettes»), qui a été également appelée dysplasie épithéliale intestinale. Nous avons identifié neuf cas de diarrhée néonatale sévère qui différaient nettement d'une atrophie microvillositaire [11]. Des explorations plus approfondies chez ces patients ont montré que la dysplasie épithéliale intestinale était un trouble épithélial constitutionel atteignant à la fois l'intestin grêle et le côlon [32]. Selon notre expérience, la dysplasie épithéliale intestinale semble fréquente chez les patients d'origine arabe, au Moyen-Orient et en Afrique du Nord. L'une des principales caractéristiques de cette maladie est son hétérogénéité clinique et histologique et son association à des malformations ou d'autres affections épithéliales.

\section{Expression clinique}

Les patients présentent typiquement une diarrhée sévère au cours des premières semaines de la vie. La plupart ont des parents consanguins et/ou un frère ou une sœur atteint. Certains membres atteints de la famille décèdent à l'âge de quelques mois des suites d'une diarrhée sévère d'origine inconnue. La diarrhée persiste le plus souvent malgré un repos intestinal, mais la mortalité est moindre que celle observée dans l'atrophie microvillositaire. Des tentatives de nutrition entérale continues à l'hydrolysat de protéines ou aux acides aminés l'aggravent. Un arrêt de croissance et une malnutrition protéinoénergétique sévère apparaissent rapidement chez ces nouveau-nés.

\section{Présentation histologique}

Une atrophie villositaire est présente, mais sa sévérité est variable. Dans sa forme typique, les anomalies sont principalement localisées à l'épithélium présentant une désorganisation des entérocytes superficiels avec des tassements focaux ressemblant à des touffes (fig. 3). Ces «houpettes» caractéristiques d'épithélium extrudé, décrites pour la première fois par Reifen et coll. [31], s'observent vers la pointe des villosités et peuvent affecter jusqu'à $70 \%$ d'entre elles. Ce processus ne se limite pas à l'intestin grêle, mais atteint également la muqueuse colique [11]. Cette image peut également s'observer dans l'épithélium des cryptes dont l'aspect est souvent anormal avec des dilatations telles que des pseudokystes et une régénérescence anormale avec ramifications [11]. L'étude des composants de la membrane basale y a mis en évidence un dépôt anormal de laminine et d'un protéoglycan à sulfate d'héparan par rapport à des échantillons de biopsies provenant de patients atteints d'une maladie coeliaque ou d'une entéropathie auto-immune [11]. Par rapport aux témoins, un dépôt très discret et irrégulier de laminine est présent à l'interface épithéliale de la lamina propria, tandis que les dépôts de protéoglycan à sulfate d'héparan sont volumineux et lamellaires, évoquant une anomalie du développement de la membrane basale. Ces dépôts pourraient être à l'origine des anomalies épithéliales. En outre, nous avons observé une augmentation de l'expression immunohistochimique de la desmogléine dans la dysplasie épithéliale intestinale, et des modifications ultrastructurales des desmosomes, dont le nombre et la longueur étaient augmentés [32] (fig. 4).

La dysplasie épithéliale intestinale est le plus souvent suspectée après qu'une atrophie microvillositaire a été exclue par l'examen de coupes après coloration PAS. Son diagnostic définitif est posé après des biopsies intestinales réitérées, dont l'aspect est subnormal au début de la vie 
Fig. 3. Dysplasie épithéliale intestinale. Atrophie villositaire partielle avec hyperplasie et/ou aspect pseudokystique des cryptes, images de ramifications et désorganisation de l'épithélium superficiel.

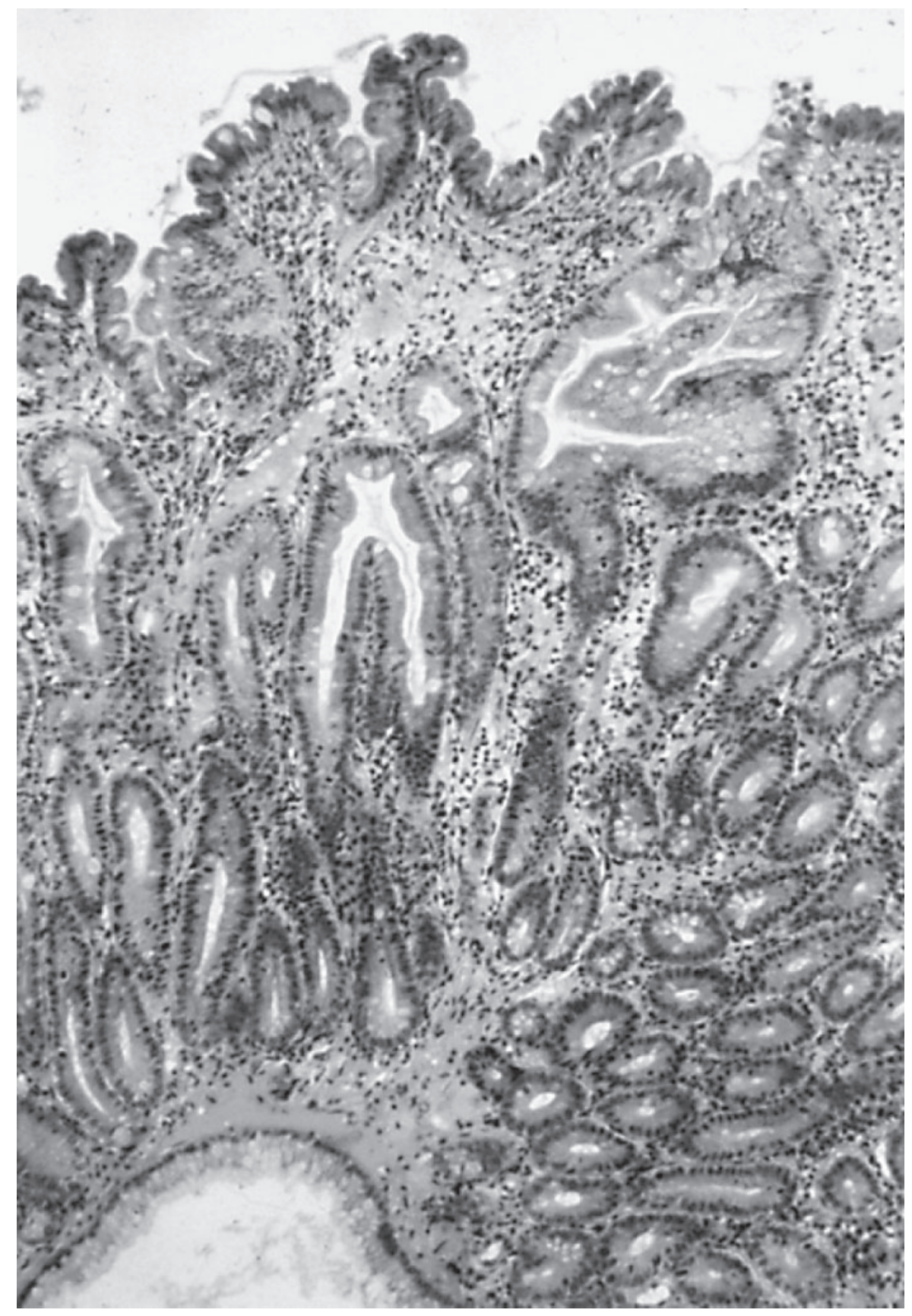

(l'atrophie villositaire non spécifique est isolée avec ou sans infiltration de la lamina propria par des cellules mononuclées) et se modifie jusqu'à l'apparition des touffes caractéristiques. Une autre difficulté est liée à l'infiltration de la lamina propria par des lymphocytes T. Ces infiltrats sont en faveur d'une entéropathie d'origine immunitaire, comme précédemment décrit particulièrement en l'absence de houpettes. Il est possible qu'un défaut de l'adhésion cellulaire augmente la perméabilité intestinale et qu'une réaction inflammatoire survienne secondairement. Murch et coll. [33] ont décrit ce type de lésion chez des nourrissons atteints d'une dysplasie intestinale.

Troubles associés

Plusieurs publications ont décrit des cas de dysplasie épithéliale intestinale associée à des anomalies phénoty- 


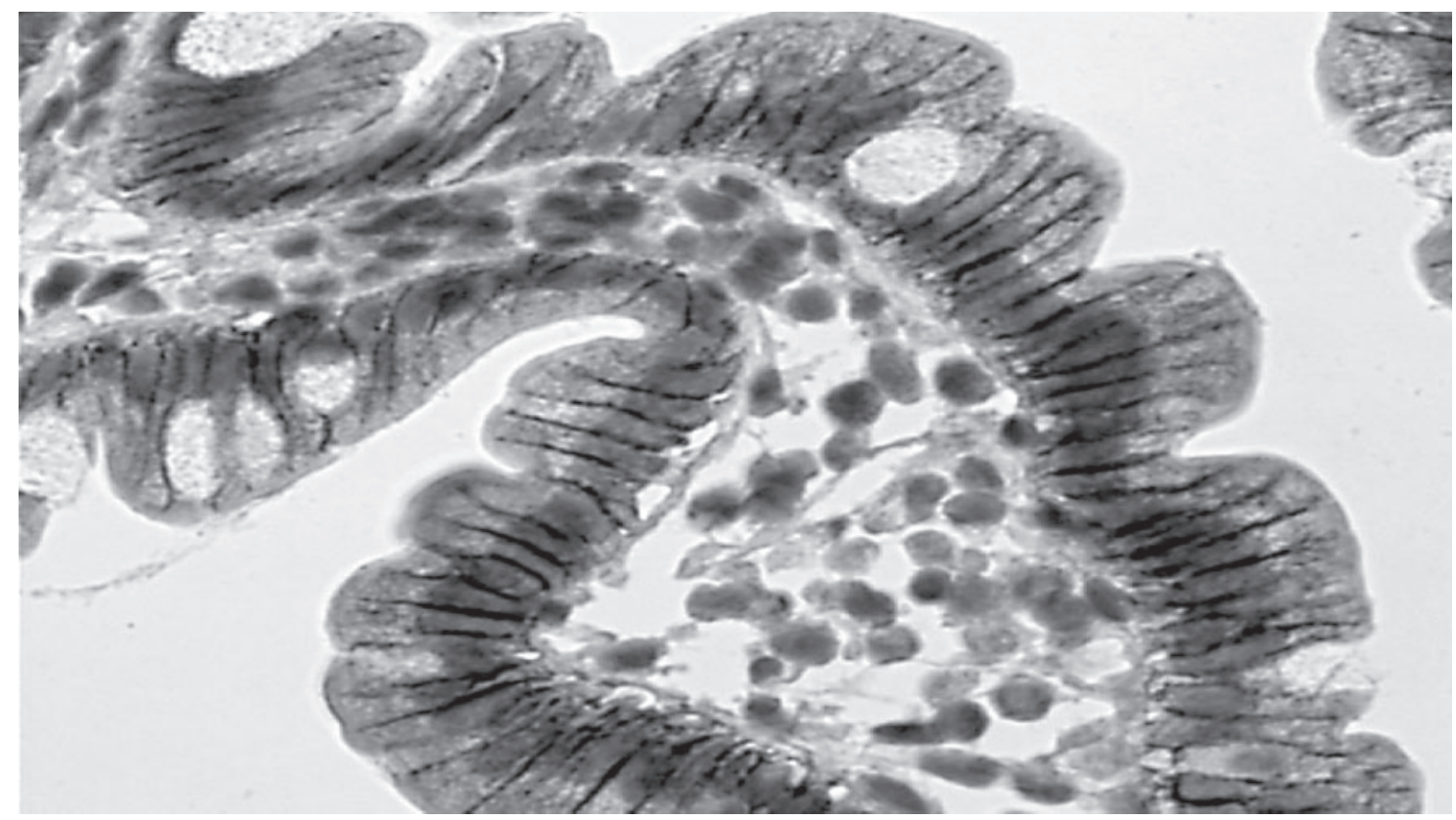

Fig. 4. Dysplasie épithéliale intestinale. Augmentation du marquage de la desmogléine des jonctions serrées chez un patient atteint d'une dysplasie épithéliale intestinale.

piques spécifiques tel qu'un syndrome de Dubowitz ou des malformations diverses [34, 35]. L'association entre une diarrhée intraitable congénitale du nourrisson et une atrésie choanale a été récemment rapportée chez quatre enfants [34]. Nous avons observé des malformations, dont une atrésie rectale ou œsophagienne, et récemment décrit une kératite ponctiforme non spécifique atteignant environ $60 \%$ des patients [36]. Cette kératite est très intrigante, car il s'agit également d'une affection épithéliale qui pourrait aider à élucider les mécanismes moléculaires de l'atteinte épithéliale intestinale. L'absence de troubles ophtalmologiques chez certains patients illustre l'hétérogénéité probable de la maladie.

Il est intéressant de noter que Lachaux et coll. [37] ont récemment décrit le cas d'un nourrisson présentant une atrésie du pylore et une diarrhée intraitable. Un examen en microscopie optique a montré une desquamation étendue du fundus au rectum, seules quelques cellules épithéliales demeurant au fond des cryptes. L'examen en microscopie électronique de l'épithélium intestinal a révélé des desmosomes normaux, mais un décollement du pôle basal des entérocytes de la lamina propria. Cette anomalie était liée à un déficit congénital en $\alpha 6 \beta 4$ intégrine, anormale dans l'épidermolyse bulleuse.
Physiopathologie et mode de transmission

Une étude a montré que dans la dysplasie épithéliale intestinale la membrane basale était anormale par rapport à celle de patients atteints d'une maladie cœliaque ou d'une entéropathie auto-immune [11]. Des molécules de la membrane basale interviennent dans les interactions entre les cellules mésenchymateuses et épithéliales qui contribuent au développement et à la différenciation intestinale [38]. Des altérations évoquant une anomalie des interactions intercellulaires et entre cellules et matrice ont été observées chez des patients atteints de dysplasie épithéliale sans aucun signe d'anomalie de la polarisation et de la prolifération des cellules épithéliales [32]. Il s'agissait d'une répartition anormale d'une molécule d'adhésion, l' $\alpha 2 \beta 1$ intégrine, le long de l'axe crypto-villositaire. L' $\alpha 2 \beta 1$ intégrine intervient dans les interactions des cellules épithéliales avec divers composants de la membrane basale tels que la laminine et le collagène. Les mécanismes physiopathologiques responsables de l'accroissement de l'expression immunohistochimique de la desmogléine et des modifications ultrastructurales des desmosomes demeurent mal compris [32]. Chez la souris, une perte de fonction du gène codant pour le facteur Elf3 est associée à des aspects morphologiques ressemblant à ceux de la dysplasie épithéliale chez le nourrisson [39]. 
Dans ce modèle murin, la morphogenèse des villosités est anormale et les cellules souches des cryptes paraissent normales. Les entérocytes de la souris déficitaire en Elf3 expriment faiblement le récepteur du facteur de croissance transformant $\beta 2$ qui induit la différenciation des épithéliums intestinaux immatures. La nature primitive ou secondaire des modifications observées chez le nourrisson reste à déterminer. La présence de houpettes pourrait constituer un indice important. Les houpettes correspondent à des cellules épithéliales non apoptotiques présentes au sommet des villosités et qui ne sont plus en contact avec la membrane basale. Bien qu'hypothétique, une anomalie de l'apoptose d'entérocytes normaux à la fin de leur durée de vie ou une altération du contact intercellulaire pourrait être responsable de cet effet. La nature primitive ou secondaire de la formation des houpettes reste à déterminer.

A ce jour, l'origine génétique de ces troubles est vraissemblable, compte tenu de la fréquence de la consanguinité parentale et/ou d'une atteinte de la fratrie qui suggèrent une transmission autosomique récessive. Le gène impliqué dans cette maladie congénitale n'est pas encore identifié. Cette entéropathie paraît plus fréquente que l'atrophie microvillositaire, particulièrement dans la population du Moyen-Orient.

\section{Évolution}

Une diarrhée néonatale qui résiste à tous les traitements nécessite une nutrition parentérale permanente. Le phénotype semble toutefois plus bénin chez certains nourrissons que chez d'autres [40]. Une fonction intestinale résiduelle et un débit de selles réduit, permettent à certains patients de ne dépendre que d'une nutrition parentérale partielle à long terme par perfusions trois ou quatre fois par semaine. Une surveillance étroite est toutefois nécessaire pour éviter l'apparition progressive d'un retard de croissance. Chez la plupart des patients, la sévérité de la malabsorption intestinale et de la diarrhée entraîne la dépendance totale d'une nutrition parentérale quotidienne à long terme avec son risque consécutif de complications. La dysplasie épithéliale intestinale peut être une indication de la transplantation intestinale [41, 42].

\section{Autres entéropathies congénitales}

La diarrhée dite «phénotypique», qui est un syndrome associant une diarrhée intraitable du nourrisson, des anomalies phénotypiques et un déficit immunitaire, est une autre maladie rare récemment décrite [12]. Les patients atteints présentent une diarrhée au cours des six premiers mois de la vie (à moins d'un mois dans la plupart des cas) et partagent plusieurs caractéristiques cliniques. Ils ont présenté un retard de croissance intra-utérin et ont une dysmorphie faciale avec front proéminent, nez épaté et hypertélorisme [12]. Ils ont aussi une trichorrhexie noueuse, anomalie dans laquelle les poils et cheveux sont laineux, difficiles à maîtriser, s'arrachent facilement et sont mal pigmentés. Ces anomalies s'observent également chez des enfants originaires du Moyen-Orient. Parmi les formes congénitales de dysplasie pileuse, la trichorrhexie noueuse est très fréquente et se voit dans plusieurs situations pathologiques $[43,44]$. Les réponses anticorps sont également défectueuses chez ces patients, malgré un taux d'immunoglobulines normal, et il en est de même des tests cutanés spécifiques d'antigènes malgré une positivité des réponses prolifératives in vitro [12]. Des échantillons de biopsies d'intestin grêle provenant de patients présentant une diarrhée syndromique montrent une atrophie villositaire modérée à sévère avec infiltrat variable de cellules mononucléées dans la lamina propria et l'absence d'anomalies épithéliales. Aucune anomalie histologique spécifique n'est présente. Le pronostic de ce type de diarrhée intraitable du nourrisson est mauvais, car la plupart des patients décèdent à l'âge de 2 à 5 ans. Certains souffrent d'une hépatopathie à début précoce [12]. La cause de cette diarrhée est inconnue et les relations entre le petit poids de naissance, la dysmorphie, la diarrhée sévère, la trichorrhexie et le déficit immunitaire ne sont pas comprises.

\section{Prise en charge de l'insuffisance intestinale}

\section{Nutrition parentérale à long terme}

En milieu hospitalier comme à domicile, une nutrition parentérale au long cours expose le malade à plusieurs types de complications, comme une septicémie et une thrombose liées au cathéter et des atteintes hépatiques et osseuses [45]. Les soins quotidiens du cathéter et la prévention des complications septiques sont essentiels [46]. Des explorations fonctionnelles hépatiques doivent être régulièrement effectuées en raison du risque de cholestase et de lésion hépatique. Une échographie destinée à identifier une boue biliaire ou une lithiase des voies biliaires doit être effectuée au moins deux fois par an chez les malades qui ont toujours leur vésicule biliaire. De plus, la minéralisation osseuse doit être surveillée par DEXA, le statut phosphocalcique doit être déterminé et la prise en charge nutritionnelle doit veiller à prévenir une ostéopathie liée à la nutrition parentérale. 
Facteurs et prise en charge d'une hépatopathie

La survenue d'une hépatopathie est la complication la plus fréquente et la plus sévère chez les patients atteints d'une insuffisance intestinale. L'expression «hépatopathie liée à la nutrition parentérale» devrait être remplacée par «hépatopathie liée à l'insuffisance intestinale». En effet, les facteurs les plus importants induisant une cholestase et une hépatopathie terminale sont liés à l'altération de la fonction intestinale et à une prolifération bactérienne excessive. Une nutrition parentérale inadéquate aggrave encore l'atteinte hépatique.

Les principaux facteurs conduisant à l'hépatopathie sont les suivants:

(1) La rupture du cycle entérohépatique (atteinte ou résection de l'iléon) [47, 48].

(2) La stase intestinale résultant en une prolifération excessive et/ou en une translocation (endotoxinémie) des bactéries intraluminales [49].

(3) Les épisodes récidivants de septicémie liée au cathéter [50, 51].

(4) La prématurité [51].

(5) Les apports inadéquats en macronutriments, comme l'apport excessif de glucose en cas de nutrition parentérale continue responsable d'un hyperinsulinisme et d'une stéatose [52] ou comme les apports inadéquats en acide aminé [49].

(6) Les apports excessifs en acides gras polyinsaturés à longue chaîne avec lipoperoxydation secondaire $[53,54]$ et la teneur élevée en phytostérols des émulsions à base d'huile de soja [55].

(7) La surcharge en aluminium, en fer et/ou en chrome [56- 58].

Les nourrissons nés avant terme ou ayant présenté un retard de croissance intra-utérin et atteints d'une entérocolite nécrosante sévère sont exposés à un risque particulièrement élevé d'hépatopathie et d'insuffisance hépatique terminale précoce dû à l'association d'une prématurité, d'une subocclusion, de septicemie à germes Gram négatif, d'un repos intestinal prolongé, d'une nutrition parentérale par perfusion continue plutôt que cyclique et d'épisodes récidivants de septicemie liée au cathéter.

La prévention ou la guérison de l'atteinte hépatique est possible grâce à:

(1) La stimulation de l'axe entérobiliaire en assurant l'ingestion de triglycérides à longues chaînes ou de lait de mère, ou par injection d'analogues de la cholécystokinine [59].

(2) La suppression de la prolifération bactérienne intraluminale excessive due à la stase intestinale par admi- nistration de métronidazole [60] et/ou la réalisation d'une entéroplastie de réduction.

(3) L'administration d'acide ursodésoxycholique (30 mg/kg/jour) afin de réduire l'atteinte hépatique [61].

(4) La prévention des septicémies liées au cathéter par des soins quotidiens appropriés de la voie veineuse [50, $51]$.

(5) L'adaptation des apports en nutrition parentérale par:

(a) La limitation des apports en glucose afin de réduire l'accumulation hépatique de lipides [62,63], et

(b) L'utilisation du type et de la quantité appropriés d'émulsion lipidique intraveineuse pour apporter les acides gras essentiels, réduire la charge en glucose et limiter la peroxydation [53-55]. Des émulsions lipidiques à base d'huile d'olive récemment développées peuvent réduire la peroxydation [54]. La quantité quotidiennne de lipides administrés par voie intraveineuse ne doit pas excéder $2-2,5 \mathrm{~g} / \mathrm{kg}$.

(6) Au contrôle des apports en lipides et du débit de leur administration et/ou à l'arrêt des lipides intraveineux dès la survenue d'une thrombopénie, d'une hyperbilirubinémie et/ou d'un ictère [53].

(7) L'utilisation des nouvelles solutions d'acides aminés adaptées à l'utilisation pédiatrique, qui apportent les acides aminés adéquats plus de la taurine [49].

(8) La cyclisation de la nutrition parentérale qui réduit l'hyperinsulinisme et la stéatose hépatique [64].

(9) L'adaptation de l'apport en fer et la diminution de la teneur en aluminium des solutions de nutrition parentérale [65].

Avec des soins et une prévention appropriés, la survenue d'une hépatopathie peut être retardée ou même évitée, et le patient peut être maintenu dans une situation nutritionnelle et générale permettant un meilleur pronostic quand une transplantation isolée de l'intestin grêle est nécessaire en raison d'une insuffisance intestinale irréversible.

\section{Transplantation intestinale}

Quelle que soit la cause d'une insuffisance intestinale prolongée ou irréversible, une nutrition parentérale prolongée est le pilier du traitement. La nutrition parentérale à domicile permet aux nourrissons et aux enfants de grandir dans le meilleur environnement psychologique possible [66, 67]. L'irréversibilité de l'insuffisance intestinale doit être démontrée avant qu'une transplantation intestinale ne soit envisagée. 
Quand vient l'heure de la transplantation intestinale, des discussions multidisciplinaires approfondies entre chirurgiens transplanteurs et gastro-entérologues pédiatres sont nécessaires. L'évaluation des risques et les décisions dépendent de la survenue des complications mentionnées au cours du séminaire qui s'est tenu lors de l'International Symposium on Small Bowel Transplantation à Miami en septembre 2003. Ces critères sont similaires à ceux présentés dans l'article de prise de position de l'American Society of Transplantation [68] et continuent à faire l'objet de débats, particulièrement ceux ayant trait aux thromboses vasculaires et aux septicémies:

(1) Hépatopathie (bilirubinémie $>1,3 \mathrm{mg} / \mathrm{dl}$, splénomégalie, thrombopénie, varices gastro-œsophagiennes, coagulopathie, saignement d'une stomie, fibrose hépatique ou cirrhose).

(2) Thrombose de l'abord veineux principal (plus de deux thromboses dans la veine sous-clavière, jugulaire ou fémorale). Dans notre pratique, un abord restant dans le lit de la veine cave supérieure.

(3) Épisodes récidivants de septicémie liée au cathéter (deux épisodes de septicémie secondaires à une infection de la ligne veineuse par an, un épisode de fongémie liée au cathéter, choc septique ou syndrome de détresse respiratoire aiguë).

(4) Maladie sous-jacente provoquant des pertes hydroélectrolytiques incontrôlables et des épisodes fréquents de déshydratation aiguë sévère.

Ces critères sont des principes directeurs et non des recommandations formelles. Ils doivent être mis en balance avec les risques liés à la transplantation intestinale.

Les contre-indications de la transplantation intestinale ne diffèrent pas de celles concernant d'autres organes solides. En raison des risques dus à la transplantation intestinale, particulièrement quand le receveur est en mauvais état général au moment de la greffe et que l'effectif des donneurs est restreint, le principe selon lequel le patient doit impérativement tirer un bénéfice manifeste de l'intervention demeure absolu.

D'autres contre-indications existent: les handicaps neurologiques congénitaux ou acquis, les affections extradigestives engageant le pronostic vital, les troubles immunitaires congénitaux ou acquis, les cancers non opérables et un abord vasculaire insuffisant pour garantir un accès veineux central facile pendant les six mois qui suivent la transplantation intestinale.

\section{Type de transplantation}

Les patients présentant une insuffisance intestinale irréversible et une hépatopathie terminale (cirrhose) sont des candidats indiscutables à des interventions destinées à éviter une issue fatale, telles la transplantation combinée du foie et l'intestin grêle. La prise en charge des malades atteints d'une fibrose hépatique sévère est plus difficile. Des biopsies hépatiques réitérées sur 6 à 12 mois et des évaluations attentives de l'hypertension portale sont nécessaires. De plus, il est difficile d'évaluer la quantité de tissu hépatique fonctionnel nécessaire pour résister à l'agression que constitue le détournement de la veine porte lors de la transplantation. Les enfants présentant une fibrose hépatique avancée sévère et progressive sont habituellement candidats à une transplantation hépato-intestinale. Certains patients dépendants de la nutrition parentérale et atteints d'une dysfonction hépatique avancée peuvent toutefois bénéficier d'une récupération hépatique fonctionnelle et biochimique se manifestant simultanément au sauvetage de l'intestin autologue. Plusieurs cas consécutifs de cholestase sévère ont totalement récupéré après l'arrêt de l'administration intraveineuse de lipides [69]. L'histologie ne permet pas toujours de prévoir la récupération fonctionnelle hépatique [70]. La survie à un an à partir de la survenue d'une cirrhose n'est toutefois que de 20 à 30\% [71].

\section{Quand adresser le patient pour une transplantation intestinale}

Les facteurs influençant la survie des enfants atteints d'une insuffisance intestinale et adressés pour transplantation intestinale sont bien connus, ce sont l'âge inférieur à un an, l'affection chirurgicale, l'existence d'une fibrose en pont ou d'une cirrhose, une bilirubinémie $>3 \mathrm{mg} / \mathrm{dl}$ et une thrombopénie [71]. Il est bien établi que les patients adressés à un centre de soins intensifs pour transplantation hépato-intestinale sont affaiblis et présentent de multiples complications, et que leur hospitalisation est de longue durée. La publication de l'UNOS indique que la mortalité des patients sur liste d'attente d'une transplantation intestinale est plus élevée que celle des patients attendant une autre transplantation [72]. Cette situation peut expliquer le faible taux de survie des patients et des greffons indiqué par diverses études [73]. Le Registre international des transplantations a confirmé que la survie à un an des malades attendant à leur domicile était meilleure que celle de ceux attendant en milieu hospitalier $(74$ contre $59 \% ; \mathrm{p}<0.00001)$ [73]. La tendance à transplanter proportionnellement plus de patients attendant à leur domicile a été un facteur qui a contribué de 
façon majeure à la récente amélioration des taux de survie des greffons et des malades [73].

Peu de centres prennent en charge tous les stades de l'insuffisance intestinale, des premières manifestations jusqu'à la transplantation intestinale, et en particulier le programme de nutrition parentérale à domicile. Il existe une importante différence d'état clinique selon que les enfants sont adressés pour transplantation intestinale par des centres disposant ou non d'équipes de soins nutritionnels [74]. La prise en charge à long terme de l'insuffisance intestinale est devenue une préoccupation très importante, comme l'ont suggéré des études récemment publiées [75]. Un réseau national ou international pourrait rassembler les données concernant l'insuffisance intestinale, données à partir desquelles les besoins de soins pourraient être evalués [76], la prise en charge optimisée, l'approche médicale actualisée et des recherches fondamentales et appliquées favorisées.

De fait, la prise en charge appropriée de l'insuffisance intestinale nécessite des centres de référence appliquant des stratégies thérapeutiques reposant sur une approche multidisciplinaire impliquant des gastro-entérologues pédiatriques, des nutritionnistes, des chirurgiens pédiatriques, un personnel infirmier spécialisé, des diététiciens, des travailleurs sociaux et des psychologues. Cette approche intégrée doit être adaptée à chaque type et stade d'insuffisance intestinale, et inclure une nutrition parentérale à domicile et un programme de transplantation.

\section{Conclusion}

La prise en charge précoce de l'insuffisance intestinale dans des centres de référence par une équipe multidisciplinaire est la clé de la reconnaissance de ce syndrome en temps utile, de l'amélioration de son évolution et de la réalisation d'une transplantation intestinale au moment adéquat. Comme l'a souligné une récente publication d'un groupe de travail en gastro-entérologie et nutrition pédiatriques, des recherches sont nécessaires dans tous les domaines de l'adaptation consécutive à une résection de l'intestin grêle et pour identifier les gènes en cause dans les entéropathies congénitales ou le syndrome de pseudoocclusion intestinale [77]. Il est également nécessaire d'évaluer les coûts respectifs de la nutrition parentérale à domicile au long cours et de la transplantation intestinale pour offir la meilleure qualité de vie aux enfants atteints et à leurs familles.

\section{Références}

-1 Nordgarrd I, Hansen BS, Mortensen PB: Importance of colonic support for energy absorption as small-bowel failure proceeds. Am J Clin Nutr 1996;64:222-231.

$\checkmark 2$ Crenn P, Coudray-Lucas C, Thuillier F, Cynober L, Messing B: Postabsorptive plasma citrulline concentration is a marker of absorptive enterocyte mass and intestinal failure in humans. Gastroenterology 2000;119:14961505.

3 Crenn P, Vahedi K, Lavergne-Slove A, Cynober L, Matuchansky C, Messing B: Plasma citrulline: A marker of enterocyte mass in villous atrophy-associated small bowel disease. Gastroenterology 2003;124:1210-1219.

-4 Pappas PA, Saudubray JM, Tzakis AG, et al: Serum citrulline and rejection in small bowel transplantation: a preliminary report. Transplantation 2001;72:1212-1216.

5 Dabbas-Tyan M, Colomb V, Rosilio M, Landais P, Ricour C, Goulet O: Evaluation of the effect of recombinant human growth hormone (rhGH) treatment of children with short bowel syndrome. J Pediatr Gastroenterol Nutr 2000; 31:S165-S166.

-6 Walker-Smith JA: Diarrhoeal disease: current concepts and future challenges. Malnutrition and infection. Trans R Soc Trop Med Hyg 1993;87(suppl 3):13-15.
7 Goulet O, Révillon Y, Jan D, et al: Neonatal short bowel syndrome. J Pediatr 1991;119:1823.

$>8$ Goulet O, Ruemmele F, Lacaille F, Colomb V: Irreversible intestinal failure. J Pediatr Gastroenterol Nutr 2004;38:250-269.

$>9$ Cuenod B, Brousse N, Goulet O, et al: Classification of intractable diarrhea in infancy using clinical and immunohistological criteria. Gastroenterology 1990;99:1037-1043.

10 Goulet O, Brousse N, Canioni D, et al: Syndrome of intractable diarrhoea with persistent villous atrophy in early childhood: A clinicopathological survey of 47 cases. J Pediatr Gastroenterol Nutr 1998;26:151-161.

11 Goulet O, Kedinger M, Brousse N, et al: Intractable diarrhea of infancy: a new entity with epithelial and basement membrane abnormalities. J Pediatr 1995; 127:212-219.

12 Giraut D, Goulet O, Ledeist F, et al: Intractable diarrhea syndrome associated with phenotypic abnormalities and immune deficiency. J Pediatr 1994; 125:36-42.

13 Davidson GP, Cutz E, Hamilton JR, Gall DG: Familial enteropathy: a syndrome of protracted diarrhea from birth, failure to thrive, and hypoplastic villous atrophy. Gastroenterology 1978;75:783-790.
14 Phillips AD, Schmitz J: Familial microvillous atrophy: A clinicopathological survey of 23 cases. J Pediatr Gastroenterol Nutr 1992;14: 380-396.

15 Holmberg C: Congenital chloride diarrhea. Clin Gastroenterol 1980;3:583-602.

16 Booth IW, Stange G, Murer H, Fenton TR, Milla PJ: Defective jejunal brush-border $\mathrm{Na}+$ / $\mathrm{H}+$ exchange: a cause of congenital secretory diarrhoea. Lancet 1985; i:1066-1069.

17 Phillips AD, Jenkins P, Raafat F, WalkerSmith JA: Congenital microvillous atrophy: specific diagnostic features. Arch Dis Child 1985;60:135-140.

18 Phillips AD, Szfranski M, Man L-Y, Wall W: Periodic acid Schiff staining abnormality in microvillous atrophy: photometric and ultrastructural studies. J Pediatr Gastroenterol Nutr 2000;30:34-42.

$\checkmark 19$ Phillips A, Fransen J, Hauri HP, Sterchi E: The constitutive exocytotic pathway in microvillous atrophy. J Pediatr Gastroenterol Nutr 1993; 17:239-246.

20 Groisman GM, Amar M, Livne E: CD10: A valuable tool for the light microscopic diagnosis of microvillous inclusion disease (familial microvillous atrophy). Am J Surg Pathol 2002; 26:902-907. 
21 Youssef N, Canioni D, Ruemmele F, et al: CD10 expression in microvillous inclusion disease (abstract). J Pediatr Gastroenterol Nutr 2003; 36:563.

-22 Raafat F, Green NJ, Nathavitharana KA, Booth IW: Intestinal microvillous dystrophy: a variant of microvillous inclusion disease or a new entity? Hum Pathol 1994;25:1243-1248.

-23 Phillips AD, Brown A, Hicks S, Schuller S, Murch SH, Walker-Smith JA, Swallow DM: Acetylated sialic acid residues and blood group antigens localise within the epithelium in microvillous atrophy indicating internal accumulation of the glycocalyx. Gut 2004;53:17641771.

-24 Nathavitharana KA, Green NJ, Raafat F, Booth IW: Siblings with microvillous inclusion disease. Arch Dis Child 1994;71:71-73.

-25 Heinz-Erian P, Schmidt H, Le Merrer M, et al: Congenital microvillous atrophy in a girl with autosomal dominant hypochondroplasia. J Pediatr Gastroenterol Nutr 1999;28:203-205.

-26 Oliva MM, Perman JA, Saavedra JM, et al: Successful intestinal transplantation for microvillous inclusion disease. Gastroenterology 1994; 106:771-774.

- 27 Herzog D, Atkinson P, Grant D, et al: Combined bowel-liver transplantation in an infant with microvillous inclusion disease. J Pediatr Gastroenterol Nutr 1996;22:405-408.

-28 Randak C, Langnas AN, Kaufman SS, et al: Pretransplant management and small bowelliver transplantation in an infant with microvillous inclusion disease. J Pediatr Gastroenterol Nutr 1998;27:333-337.

29 Ruemmele FM, Jan D, Lacaille F, et al: New perspectives for children with microvillous inclusion disease: early small bowel transplantation. Transplantation 2004;77:1024-1028.

-30 Croft NM, Howatson AG, Ling SC, et al: Microvillous inclusion disease: an evolving condition. J Pediatr Gastroenterol Nutr 2000;31: 185-189.

- 31 Reifen RM, Cutz E, Griffiths AM, Ngan BY, Sherman PM: Tufting enteropathy: a newly recognized clinicopathological entity associated with refractory diarrhea in infants. J Pediatr Gastroenterol Nutr 1994;18:379-385.

32 Patey N, Scoazec JY, Cuenod-Jabri B, et al: Distribution of cell adhesion molecules in infants with intestinal epithelial dysplasia (tufting enteropathy). Gastroenterology 1997;113: 833-843.

- 33 Murch S, Graham A, Vermault A, et al: Functionally significant secondary inflammation occurs in a primary epithelial enteropathy. J Pediatr Gastroenterol Nutr 1997;24:467.

- 34 Krantz M, Jansson U, Rectors S, Ryd W, Kristiansson B: Hereditary intractable diarrhea with choanal atresia. A new familial syndrome. J Pediatr Gastroenterol Nutr 1997;24:470.

35 Abely M, Fromont Hankard G, et al: Intractable infant diarrhea with epithelial dysplasia associated with polymalformation. J Pediatr Gastroenterol Nutr 1998;27:348-352.
36 Djeddi D, Verkarre V, Talbotec C, et al: Tufting enteropathy and associated disorders (abstract). J Pediatr Gastroenterol Nutr 2002;34: 446.

37 Lachaux A, Bouvier R, Loras I, et al: Isolated deficient $\alpha 6 \beta 4$ integrin expression in the gut associated with intractable diarrhoea. J Pediatr Gastroenterol Nutr 1999;29:395-401.

38 Simon-Assmann P, Kedinger M: Heterotypic cellular cooperation in gut morphogenesis and differentiation. Cell Biol 1993;4:221-230.

39 Ayn NG, Waring P, Ristevski S, et al: Inactivation of the transcription factor Elf3 in mice results in dysmorphogenesis and altered differentiation of intestinal epithelium. Gastroenterology 2002;122:1455-1466.

40 Cameron DJS, Barnes GL: Successful pregnancy outcome in tufting enteropathy. J Pediatr Gastroenterol Nutr 2003;36:158

41 Lacaille F, Cuenod B, Colomb V, et al: Successful combined liver and small bowel transplantation in a child with epithelial dysplasia. J Pediatr Gastroenterol Nutr 1998;27:230-233.

42 Paramesh AS, Fishbein T, Tschernia A, et al: Isolated small bowel transplantation for tufting enteropathy. J Pediatr Gastroenterol Nutr 2003;36:138-140.

43 Itin PH, Pittelkow MR: Trichothiodystrophy: review of sulfur-deficient brittle hair syndromes and association with the ectodermal dysplasia. J Am Acad Dermatol 1990;22:705717.

44 Verloes A, Lombet J, Lambert Y, et al: Trichohepato-enteric syndrome: further delineation of a distinct syndrome with neonatal hemochromatosis phenotype, intractable diarrhea, and hair anomalies. Am J Med Genet 1997;68 391-395.

-45 Shulman RJ, Phillips S: Parenteral nutrition in infants and children. J Pediatr Gastroenterol Nutr 2003;36:587-607.

- 46 Colomb V, Fabeiro M, Dabbas M, Goulet O, Merckx J, Ricour C: Central venous catheterrelated infections in children on long-term home parenteral nutrition: incidence and risk factors. Clin Nutr 2000;19:355-359.

47 Colomb V, Goulet O, Rambaud C, et al: Long term parenteral nutrition in children: liver and gallbladder disease. Transplant Proc 1992;24: 1054-1055.

48 Moss RL, Das JB, Raffensperger JG: Total parenteral nutrition-associated cholestasis: clinical and histopathological correlation. J Pediatr Surg 1993;28:1270-1275.

49 Forchielli ML, Walker WA: Nutritional factors contributing to the development of cholestasis during total parenteral nutrition. Dev Pediatr 2003;50:245-267.

50 Colomb V, Jobert-Giraud A, Lacaille F, Goulet O, Fournet JC, Ricour C: Role of lipid emulsions in cholestasis associated with long-term parenteral nutrition in children. J Parenter Enteral Nutr 2000;24:345-350.

51 Moseley RH: Sepsis-associated cholestasis. Gastroenterology 1997;112:302-305.
52 Lienhardt A, Rakotoambinina B, Colomb V, et al: Insulin secretion and sensitivity in children on cyclic total parenteral nutrition. J Parenter Enteral Nutr 1998;22:382-386.

53 Belli DC, Fournier LA, Lepage G, et al: Total parenteral nutrition-associated cholestasis in rats: comparison of different amino acid mixtures. J Parenter Enteral Nutr 1987;11:6773.

54 Goulet $\mathrm{O}$, de Potter S, Antebi H, et al: Longterm efficacy and safety of a new olive oil-based intravenous fat emulsion in pediatric patients: a double-blind randomized study. Am J Clin Nutr 1999;70:338-345.

55 Clayton PT, Whitfield P, Iyer K: The role of phytosterols in the pathogenesis of liver complications of pediatric parenteral nutrition. Nutrition 1998:14:158-164.

56 Popinska K, Kierkus J, Lyszkowska M, et al: Aluminum contamination of parenteral nutrition additives, amino acid solutions, and lipid emulsions. Nutrition 1999;15:683-686.

$\checkmark 57$ Ben Hariz M, Goulet O, De Potter S, et al: Iron overload in children receiving prolonged parenteral nutrition. J Pediatr 1993;123:238241.

58 Moukarzel A, Song MK, Buchman AL, et al: Excessive chromium intake in children receiving total parenteral nutrition. Lancet 1992; 339:385-388.

59 Ling PR, Sheikh M, Boyce P, et al: Cholecystokinin (CCK) secretion in patients with severe short bowel syndrome (SSBS). Dig Dis Sci 2001;46:859-864.

60 Capron JP, Gineston JL, Herve MA: Metronidazole in prevention of cholestasis associated with total parenteral nutrition. Lancet 1983;i:446-447.

61 Heubi JE, Wiechmann DA, Creutzinger V, et al: Tauroursodeoxycholic acid (TUDCA) in the prevention of total parenteral nutrition-associated liver disease. J Pediatr 2002;141:237242.

62 Bresson JL, Narcy P, Putet G, et al: Energy substrate utilization in infants receiving total parenteral nutrition with different glucose to fat ratios. Pediatr Res 1989;25:645-648.

63 Kaufman SS: Prevention of parenteral nutrition-associated liver disease in children. Pediatr Transplant 2002;6:37-42.

64 MacFie J: Cyclic parenteral nutrition. Nutrition 1997;13:46-48.

65 Advenier E, Landry C, Colomb V, et al: Aluminum contamination of parenteral nutrition and aluminum loading in children on longterm parenteral nutrition. J Pediatr Gastroenterol Nutr 2003;36:448-453.

66 Colomb V, Goulet O, Ricour C: Home enteral and parenteral nutrition: Baillieres Clin Gastroenterol 1998;122:877-894.

67 Scolapio JS: A review of the trends in the use of enteral and parenteral nutrition support. J Clin Gastroenterol 2004;38:403-407.

68 Kaufman S, Atkinson JB, Bianchi A, Goulet O, Grant D, Langnas A, et al: Indications for pediatric intestinal transplantation: A position paper of the American Society of Transplantation. Pediatr Transplant 2001;5:80-87. 
-69 Colomb V, Jobert-Giraud A, Lacaille F, Goulet O, Fournet JC, Ricour C: Role of lipid emulsions in cholestasis associated with long-term parenteral nutrition in children. JPEN J Parenter Enteral Nutr 2000;24:345-350.

70 Iyer K, Horslen S, Torres C, et al: Histology is not predictive of functional liver recovery in parenteral-nutrition associated liver dysfunction (abstract). Pediatr Transplant 2003;7:69.

71 Bueno J, Ohwada S, Kocoshis S, et al: Factors impacting the survival of children with intestinal failure referred for intestinal transplantation. J Pediatr Surg 1999;34:27-33.
Fryer J, Pellar S, Ormond D, Koffron A, Abecassis M: Mortality in candidates waiting for combined liver-intestine transplants exceeds that for other candidates waiting for liver transplants. Liver Transplant 2003;9:748753.

73 Grant D, Abu-Elmagd K, Reyes J, Tzakis A, Langnas A, Fishbein T, Goulet O: Farmer D, on the behalf of the Intestine Transplant Registry: 2003 report of the intestine transplant registry: a new era has dawned. Ann Surg 2005; 241:607-613.

74 Beath SV, Booth IW, Murphy MS, et al: Nutritional care and candidates for small bowel transplantation. Arch Dis Child 1995;73:348350 .

-75 Fishbein TM, Schiano T, LeLeiko N, et al: An integrated approach to intestinal failure. Results of a new program with total parenteral nutrition, bowel rehabilitation, and transplantation. J Gastrointest Surg 2002;6:554-562.
76 Guarino A, De Marco G, Italian National Network for Pediatric Intestinal Failure: Natural history of intestinal failure, investigated through a national network-based approach. J Pediatr Gastroenterol Nutr 2003;37:136141.

7 Kocoshis SA, Beath S, Booth IW, Oliva GA, Goulet O, Kaufman SS, Lai HS, Luque C, Ohtsuka Y: Intestinal failure and small bowel transplantation, including clinical nutrition: Working Group report of the second World Congress of Pediatric Gastroenterology, Hepatology and Nutrition. J Pediatr Gastroenterol Nutr 2004;39(suppl 2):S655-S661. 